\section{One-year result of XEN45 implant for glaucoma: efficacy, safety, and postoperative management} SZ $\operatorname{Tan}^{1,2}$, A Walkden ${ }^{2,3}$ and $\mathrm{L} \mathrm{Au^{2,3 }}$

\begin{abstract}
Purpose To report the efficacy, safety profile, and postoperative management of XEN45 implant at 12-month follow-up.

Patients and methods This was a retrospective, non-randomised interventional case series involving patients who underwent XEN45 implantation by a single, experienced glaucoma specialist in a tertiary centre. Primary outcome measures were the intraocular pressure (IOP) and the number of medications at 1-year follow-up visit. Two IOP criteria were chosen to measure success: IOP $\leq 21 \mathrm{~mm} \mathrm{Hg}$ and $\geq 20 \%$ reduction from baseline (criteria one); and IOP $\leq \mathbf{1 5} \mathrm{mm} \mathrm{Hg}$ and $\geq 30 \%$ reduction from baseline (criteria two).
\end{abstract}

Results All 39 eyes completed 1-year followup. The mean preoperative IOP was $24.9 \pm 7.8 \mathrm{~mm} \mathrm{Hg}$ on three drops, which reduced to $14.5 \pm 3.4 \mathrm{~mm} \mathrm{Hg}$ at month 12 $(P<0.005)$ on 0.7 drops $(P<0.005)$. On the basis of criteria one, the cumulative probability of success at 1 year was $87.0 \%$ without medication and $\mathbf{9 2 . 0 \%}$ with medication. On the basis of criteria two, cumulative probability of success was $62.0 \%$ without medication and $64.0 \%$ with medication. Bleb intervention with a median of 2 (range 1-4) was required for $51.3 \%$ of eyes. Implant was obstructed by iris tissue in three eyes $(7.7 \%)$; one eye $(2.6 \%)$ developed hyphaema; eight eyes (20.5\%) had numerical hypotony (IOP $\leq 5 \mathrm{~mm} \mathrm{Hg}$ ) at day 1 , of which all spontaneously resolved by week 4 apart from one eye.

Conclusions The XEN45 implant proved to be an effective treatment with a good safety profile at 1-year follow-up period. The high rate of postoperative bleb intervention does not make XEN45 a 'fit-and-forget' procedure and therefore the procedure should ideally be performed by surgeons experienced in bleb management.

Eye (2018) 32, 324-332; doi:10.1038/eye.2017.162; published online 1 September 2017

\section{Introduction}

Minimally invasive glaucoma surgery (MIGS) has attracted substantial interest within the glaucoma community in recent years. There has been particular interest in developing microstents, which can lower intraocular pressure (IOP) in a similar manner to traditional glaucoma drainage surgeries, but with a better safety profile and shorter surgical time. ${ }^{1}$ The iStent (Glaukos Corporation, Laguna Hills, CA, USA) and Hydrus microstent (Ivantis Inc., Irvine, CA, USA) are Schlemm's canal stents, designed to allow aqueous humour to egress into Schlemm's canal more efficiently directly from the anterior chamber (AC). Studies have shown that these stents have the potential to safely lower IOP to the $15-20 \mathrm{~mm} \mathrm{Hg}$ range in open-angle glaucoma patients. ${ }^{2-5}$ Where a lower IOP may be required due to advanced glaucomatous change, these stents may not be the intervention of choice. The suprachoroidal stents, Cypass (Transcend Medical, Menlo Park, CA, USA) and iStent Supra (Glaukos Corporation), provide direct communication between the AC and suprachoroidal space. Studies examining these stents are still limited but initial results showed a similar IOP reduction to the other stents mentioned above. ${ }^{6}$

Subconjunctival outflow has been proven to deliver the greatest IOP reduction and has been the traditional outflow pathway for glaucoma drainage surgery. XEN45 gel implant (Aquesys / Allergan Inc., Irvine, CA, USA), made of a soft, 
permanent, collagen-derived gelatin, is $6 \mathrm{~mm}$ long and with an internal lumen of $45 \mu \mathrm{m}$. It is injected ab internally, through the trabecular meshwork via a scleral tunnel into the subconjunctival/subtenon space. The implant is designed to regulate the outflow of aqueous from the AC into the non-dissected tissue of the subconjunctival space. It simulates traditional drainage surgery, but has the potential benefits of being minimally invasive and safer to perform.

The aim of this study is to report the efficacy, safety profile, and postoperative management of XEN45 implants at the Manchester Royal Eye Hospital, UK.

\section{Materials and methods}

This was a retrospective, consecutive, non-randomised interventional case series involving patients who underwent XEN45 implantation from September 2014 to February 2016.

Patients with gonioscopically confirmed open-angle glaucoma, who were taking at least one IOP-lowering medication, were included in the study. All patients with neovascular glaucoma had been treated with extensive panretinal photocoagulation before the XEN45 implant. Patients who had simultaneous cataract surgery were excluded from the study. Informed consent was obtained from all patients after explanation of the procedure and potential associated risks. All procedures followed the tenets of the Declaration of Helsinki. We certify that all applicable institutional and governmental regulations concerning the ethical use of human volunteers were followed during this research.

All cases were performed by a single, glaucoma specialist with extensive experience in traditional filtration surgery, bleb management and MIGS (LA). A standard dose of $0.2 \mathrm{mg} / \mathrm{ml}$ of mitomycin-C (MMC) was injected into the subconjunctival/subtenon space at 12 o'clock $4-5 \mathrm{~mm}$ behind the surgical limbus. It was then massaged across to the supranasal area and left for $5 \mathrm{~min}$. A self-sealing corneal wound was then made inferotemporally, and Miochol-E (Bausch and Lomb, Surrey, UK) along with Healon GV (Abbott Medical Optics, Inc., Santa Ana, CA, USA) was injected to constrict the pupil and deepen the AC. The XEN45 was implanted ab internally using a pre-loaded injector, superior to the trabecular meshwork via a scleral tunnel into the subconjunctival/subtenon space. The viscoelastic was then aspirated. Attention was paid to ensure the implant was inserted correctly, with subsequent bleb formation noted at the end of surgery. All patients received intracameral antibiotics and subconjunctival steroid injections.

Patients were advised to discontinue all glaucoma medications on day 1 and given chloramphenicol eye drops QDS, atropine 1\% OD, and prednisolone 1\% six times a day. The prednisolone $1 \%$ was tapered gradually over 3 months according to the amount of conjunctival injection postoperatively. In the uveitic glaucoma group, no extra systemic treatment was used peri-operatively but patients were advised to use the prednisolone $1 \%$ eye drop two hourly from day 1 post surgery. All patients were reviewed at 1 day, 1 week, 1 month, 3 months, 6 months, and 12 months. Further follow-ups were arranged between these dates as required.

Postoperative bleb interventions were carried out at the discretion of the same surgeon who performed the surgery. The indications for these interventions were similar to post-trabeculectomy management and decision was based on bleb morphology, conjunctival injection, and IOP. In this study, hyperaemic blebs with good IOP would receive a standard dose of $0.1 \mathrm{ml}$ of $50 \mathrm{mg} / \mathrm{ml} 5-$ fluorouracil (5-FU) injections, administered directly superior to the bleb. Encysted or encapsulated blebs with high IOP would receive bleb needling with 5-FU, often performed at the slit lamp. Continued bleb failure despite needling attempts would receive bleb revision surgery, which involved a local conjunctival peritomy initially, followed by dissection of any underlying scar tissue. The XEN45 implant was then mobilised to ensure drainage. $0.2 \mathrm{mg} / \mathrm{ml} \mathrm{MMC}$ was subsequently reapplied using three to four cut sponges under the subtenon space and the conjunctiva was then closed using 10/0 Nylon sutures. Needling with a 5-FU injection was always considered before recommencing on any IOP-lowering medication.

Patient demographics, best-corrected visual acuity (BCVA), IOP measured with Goldmann tonometry, number of glaucoma medications, type of interventions, and complications were recorded for each study visit.

Primary outcome measures were the IOP and medications at the 1-year follow-up visit. The results were presented using the World Glaucoma Association recommendations. We had specifically taken into considerations the reported outcomes from the trabeculectomy study by Kirwan et $a l^{7}$ and other MIGS studies. ${ }^{6,8,9}$ Twocmulative probability of success:

1. IOP $\leq 21 \mathrm{~mm} \mathrm{Hg}$ and $\geq 20 \%$ reduction from baseline

2. IOP $\leq 15 \mathrm{~mm} \mathrm{Hg}$ and $\geq 30 \%$ reduction from baseline

Complete success was when the IOP criteria were achieved without medications and qualified success was when IOP criteria were achieved regardless of medications. The time to failure was defined as the time from 1 month after surgery to the time where IOP was measured either below the lower limit $(<6 \mathrm{~mm} \mathrm{Hg})$ or above the upper limits described earlier on two consecutive postoperative visits. If additional drainage 
procedures were required, this was also classed as a failure. Bleb intervention was not considered as failure. In all cases, the second consecutive visit was recorded as the time of failure.

Statistical analysis was performed using Microsoft Excel (London, UK) and Xlstat 2016.1 (New York City, NY, USA). Student's $t$-test was used to compare continuous variables, and a $P<0.05$ was considered statistically significant. The cumulative probability of success was assessed using a stratified Kaplan-Meier survival analysis.

\section{Results}

\section{Baseline characteristics}

A total of 43 XEN45 procedures were performed during the study period, of which four had simultaneous cataract surgery and were therefore excluded from the study. All 39 patients (female : male $=1.2: 1$ ) completed 12-month follow-up. Mean age was 70.1 years (range 32-92). The patient demographics are presented in Table 1a.

\section{Treatment outcomes}

The mean preoperatively BCVA was $0.23 \log$ MAR, which dropped to $0.37 \log$ MAR on day 1 and $0.40 \log$ MAR at month 1 , but stabilised at $0.16 \log$ MAR by month 3 and $0.21 \log$ MAR by month 12 postoperatively. Two patients lost more than two Snellen lines at final follow-up visit, both of whom had uveitis-related complications leading to loss of vision. None of the patients developed severe postoperative inflammation.

The mean preoperative IOP was $24.9 \pm 7.8 \mathrm{~mm} \mathrm{Hg}$ on three drops, and four patients were taking acetazolamide tablets. This reduced to $9.4 \pm 5.4 \mathrm{~mm} \mathrm{Hg}$ on day 1 using

Table 1a Patient demographics

\begin{tabular}{lc}
\hline Number of cases & 39 \\
Mean age & $70.1 \pm 13.8$ (range 32-92) \\
Female : male & $1.2: 1$ \\
& $\mathrm{~N}(\%)$ \\
Type of glaucoma & $30(71.7)$ \\
$\quad$ Primary open angle & $2(5.13)$ \\
Pseudoexfoliation & $1(2.56)$ \\
Pigment dispersion syndrome & $4(10.3)$ \\
Uveitic & $1(2.56)$ \\
Rubeotic & $1(2.56)$ \\
Steroid induced & $\mathrm{N}(\%)$ \\
& $12(30.8)$ \\
Previous surgery & $3(7.7)$ \\
Cataract surgery & $2(5.13)$ \\
Trabeculectomy & \\
iStent & 0.8 (range $0.5-0.95)$ \\
Mean cup : disc & $-6.8 \pm 5.9$ \\
Mean visual field mean deviation &
\end{tabular}

no IOP-lowering medications, and $13.7 \pm 3.7 \mathrm{~mm} \mathrm{Hg}$ at month 1 using a mean of 0.1 medications. Mean IOP was $14.9 \pm 4.9 \mathrm{~mm} \mathrm{Hg}$ at month 3 on 0.1 medications, $14.2 \pm 3.9 \mathrm{~mm} \mathrm{Hg}$ at month 6 on 0.3 medications, and $14.5 \pm 3.4 \mathrm{~mm} \mathrm{Hg}$ at month $12(P<0.005)$ on 0.7 medications $(P<0.005)$ (Figures $1 \mathrm{a}$ and $\mathrm{b})$. A $41.8 \%$ IOP reduction was achieved at month 12 from baseline. IOPlowering medication was not required for $77.0 \%$ and $59.0 \%$ of eyes at 6 and 12 months, respectively.

Scatter plot in Figure 2 illustrates the preoperative IOP against the postoperative IOP at 12 months for all 39 eyes. All eyes but 1 demonstrated reduction of IOP postoperatively. The proportion of eyes that met various target IOP levels at 1 -year follow-up of IOP $\leq 21, \leq 18$, $\leq 15$, and $\leq 12 \mathrm{~mm} \mathrm{Hg}$ regardless of medication (qualified success) were $95.0 \%, 92.0 \%, 66.6 \%$, and $25.6 \%$, respectively. On the other hand, 56.4\%, 56.2\%, 51.3\%, and $25.6 \%$ of eyes, respectively, achieved those targets without medications (complete success).

A Kaplan-Meier survival curve at 1 year is demonstrated in Figure 3. On the basis of criteria one of IOP $\leq 21 \mathrm{~mm} \mathrm{Hg}$ and $\geq 20 \%$ reduction from baseline, the cumulative probability of success at 1 year was $87.0 \%$ without medications and $92.0 \%$ with medications. When IOP $\leq 15 \mathrm{~mm} \mathrm{Hg}$ and $\geq 30 \%$ reduction from baseline were used as the criteria, the cumulative probability of success was $62.0 \%$ without medications and $64.0 \%$ with medications.

\section{Postoperative interventions and complications}

Nineteen eyes (48.7\%) required no bleb intervention at all during the follow-up period. The remaining $51.3 \%$ of eyes required bleb intervention with a median number of two episodes (range 1-4). The first bleb intervention was performed as early as 1 week and as late as 5 months postoperatively (median $=4$ weeks). Two patients required bleb revision at month 1 and month 3 , respectively; one of these patients had previous combined phaco-iStent surgery and the other had steroid eye drops withdrawn prematurely at week 2 by mistake due to miscommunication. Both of them had fibrotic bleb and raised IOP. XEN45 implant was found to be obstructed by iris tissue in three eyes (7.7\%) and these were successfully treated with focal Argon laser iridoplasty. One eye (2.6\%) developed hyphaema requiring AC washout. Eight eyes (20.5\%) had numerical hypotony (IOP $\leq 5 \mathrm{~mm} \mathrm{Hg}$ ) on day 1 , of which all resolved without intervention by week 4 except for one eye, which required $\mathrm{AC}$ reformation at week 3 . One patient went on to have combined phaco-iStent after 6 months' visit but no patients required glaucoma drainage surgery at final follow-up visit. 
a
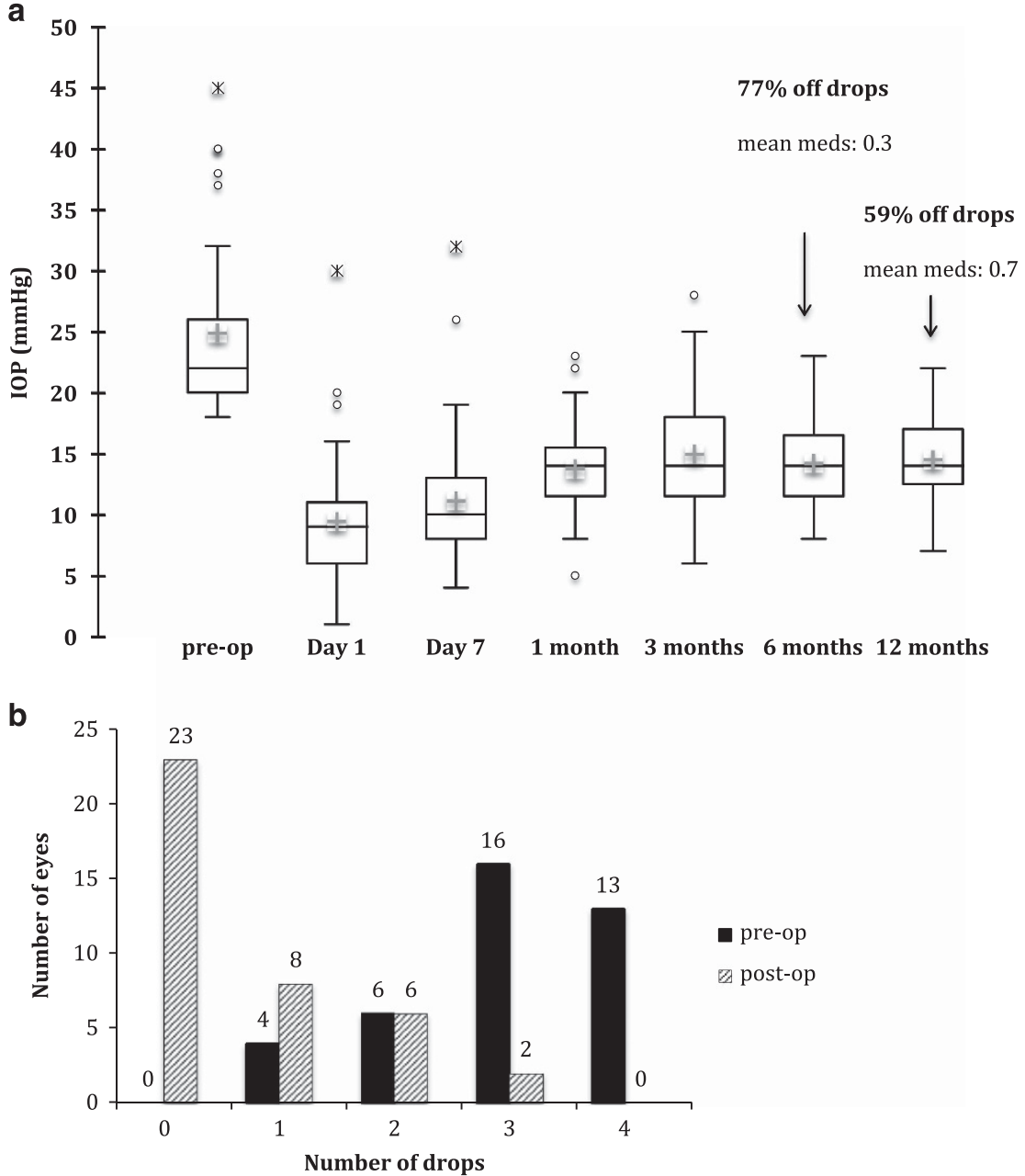

Figure 1 (a) Change in mean IOPs over 12-month follow-up visits. (b) Change in number drops at last follow-up visit from baseline.

\section{Bleb intervention vs no bleb intervention subgroup analysis}

Subgroup analysis was performed in those eyes that required bleb intervention at any point within the year vs those who did not. There were a higher number of phakic patients in the bleb intervention group. The rest of the baseline characteristics in both groups were comparable. (Table 1b).

The mean IOP and number of medications at each follow-up visits are illustrated in Figure 4. There was no significant statistical difference between the mean preoperative IOP and number of medications between the two groups. Both groups achieved a significant IOP reduction $(P<0.005)$ to $9.8 \pm 7.1$ and $9.1 \pm 3.1 \mathrm{~mm} \mathrm{Hg}$ at day 1 , respectively, implying correct implantations. However, at month 1 , the bleb intervention group experienced a significant increase in IOP to $15.3 \pm 3.4 \mathrm{~mm} \mathrm{Hg}$ from day $1(P<0.001)$. The bleb intervention group continued to have higher IOP than the non-intervention group throughout the follow-up visits and the IOP between the two groups at month 12 was statistically significant $(P=0.03)$. The number of medications between the two groups at month 12 was non-statistically significant $(P=0.36)$.

\section{Discussion}

Little published data currently exists for XEN45 implant. Pérez-Torregrosa et al ${ }^{10}$ looked at combined phaco-XEN45 augmented with MMC in 30 eyes and found a $29.3 \%$ reduction in IOP at 12-month follow-up with $94.6 \%$ drop reduction. The XEN45 phase IV APEX study is currently ongoing, evaluating the efficacy and safety of XEN alone and combined phaco-XEN surgery over a 24-month period. Although unpublished, the interim 1-year results have been presented in scientific meetings. So far the standalone XEN implant group demonstrated a $32.5 \%$ reduction in IOP at 12 months to a mean of $13.6 \mathrm{~mm} \mathrm{Hg}$ on 0.7 drops. In our study, we found a greater reduction of IOP of $41.8 \%$ at 12 months; our mean IOP at 1 year was higher at 


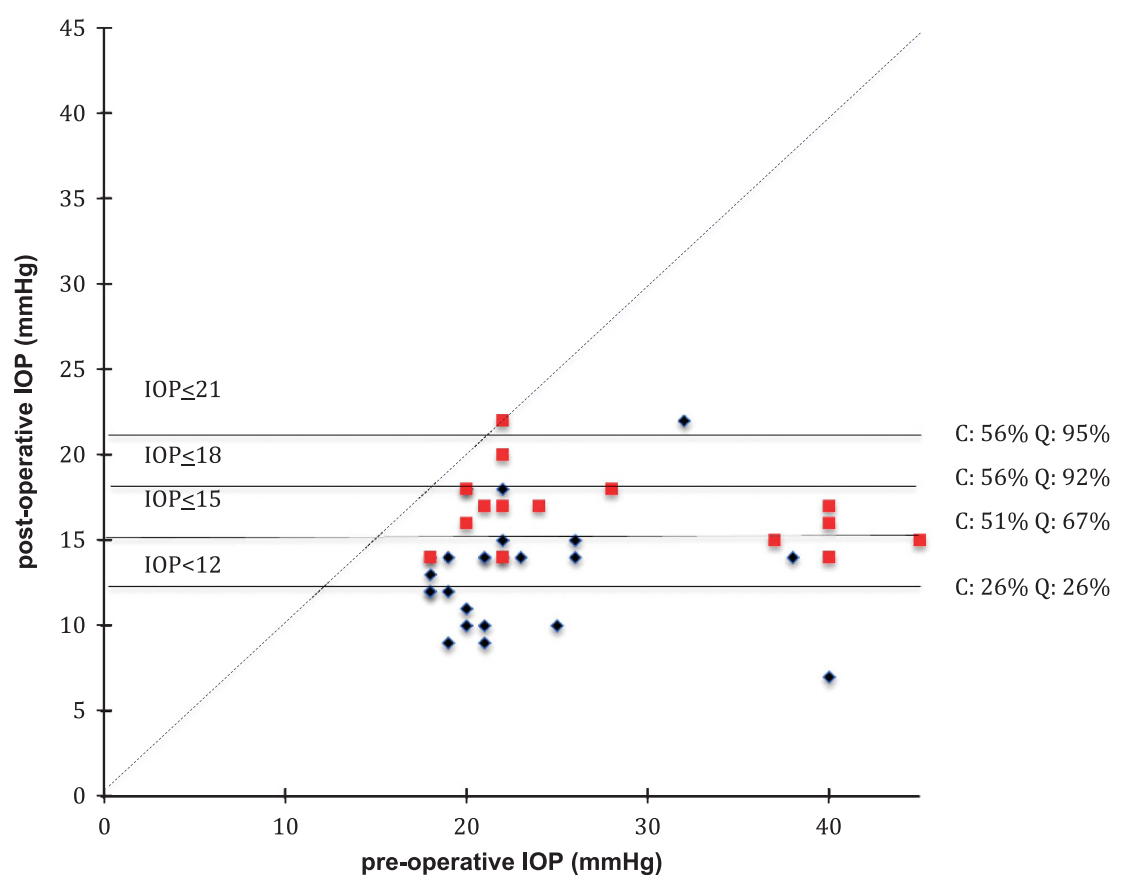

Figure 2 Scatter plot of preoperative $v$ s postoperative IOP at 1-year follow visit with lines demonstrating the proportion of eyes that met various criteria for success $(\leq 21, \leq 18, \leq 15$, and $\leq 12 \mathrm{~mm} \mathrm{Hg}$ ). C, complete success; diamond, not on drops; Q, qualified success; square, back on drops.

$14.5 \mathrm{~mm} \mathrm{Hg}$ with a similar medication count of 0.7. Our overall, medication free percentage for the whole cohort was $59.0 \%$ at 1 year compared with the APEX study, which was $55.0 \%$. Compared to other MIGS results published in the literature, single iStent insertion without cataract surgery achieved $27.0 \%$ IOP reduction at 12 months $(10.8 \%$ on IOP-lowering drops), ${ }^{11}$ double iStents achieved $36.0 \%$ IOP reduction ( $9.8 \%$ on drops) ${ }^{8} 40 \%$ with triple iStents (7.8\% on drops), ${ }^{8} 35 \%$ with Cypass on 1.4 drops, ${ }^{12}$ and $36 \%$ with trabectome on 2.6 drops. ${ }^{9}$ The Hydrus study by Gandolfi et al ${ }^{13}$ showed a $38.0 \%$ IOP reduction on 0.9 IOPlowering drops at 24 months. Direct comparison with these aforementioned studies need to be interpreted with care as they have different inclusion and exclusion criteria. In our study, the retrospective nature allowed us to include a wider range of severity; patients with advanced glaucoma, secondary open-angle glaucoma, and previous failed trabeculectomy were included, which would not have been the case for many other MIGS studies.

By using subconjunctival outflow and postoperative bleb management, XEN implant is akin to traditional trabeculectomy surgery, without requiring a scleral flap, osteum, iridectomy, or releasable sutures. When applying the strict criteria of $\leq 21 \mathrm{~mm} \mathrm{Hg}$ and $\leq 80.0 \%$ preoperative IOP, the 12-month cumulative probability of qualified and complete success were 92.0 and $87.0 \%$ in our study, respectively, compared to $\sim 90 \%$ (figure derived from graph, exact figure was not reported) in the trabeculectomy study by Kirwan et al. ${ }^{7}$ Kirwan et al ${ }^{7}$ also reported an IOP reduction of $46.0 \%$ in a multicentre retrospective study of 428 post-trabeculectomy eyes at 1 year on 0.1 medications, with a mean final IOP of $12.5 \mathrm{~mm} \mathrm{Hg}$, which is more efficacious than this XEN45 series. It is therefore reasonable to conclude that trabeculectomy is still a superior procedure in terms of successful IOP lowering. However, within the ophthalmic community, enhanced trabeculectomy remains a subspecialist procedure with surgeon-dependent factors, which can produce varied results. ${ }^{14,15}$ The study by Kirwan et al included a large number of trabeculectomies performed by an experienced group of surgeons and unsurprisingly demonstrated some of the best trabeculectomy results published in the literature. The fact that our first 39 XEN45 implants showed comparable results to trabeculectomy is very encouraging. With more clinical experience, better case selection, and refined postoperative management it would not be unreasonable to expect better outcomes in the future.

The success of the XEN45 implant in our study was partly attributable to the intensive postoperative bleb management. Compared to a $32.1 \%$ needling rate in the unpublished APEX study, 51.3\% of eyes in our study required bleb intervention from as early as 1 week to 5 months postoperatively. Bleb needling and 5-FU injection were found to be required in $17.0 \%$ and $28.0 \%$ of post-trabeculectomy eyes, respectively. ${ }^{7}$ Our bleb intervention rate was high, and indeed higher than our own current practise for post-trabeculectomy eyes 

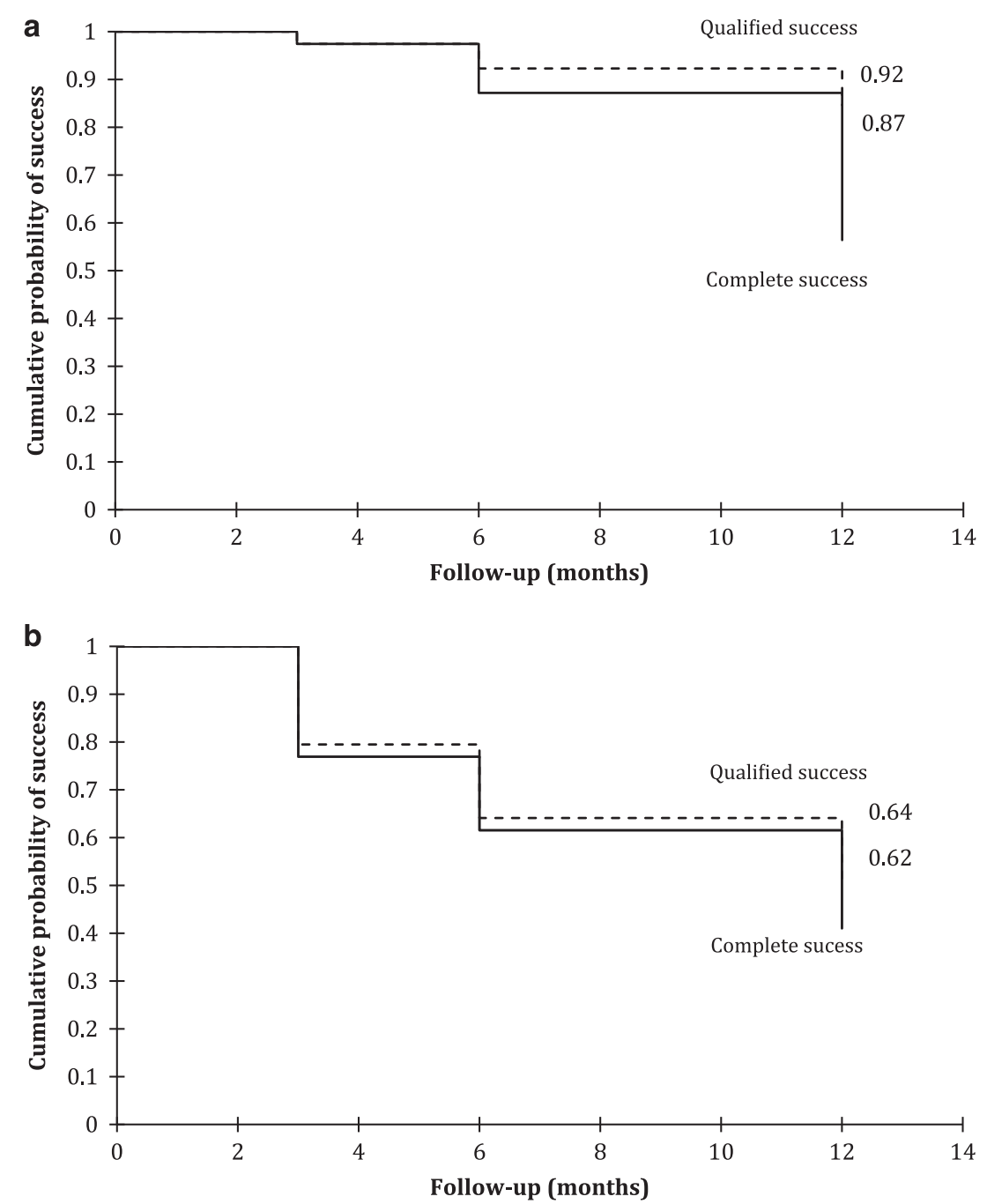

Figure 3 Kaplan-Meier survival curve at 12 months, defining failure as (a) IOP $>21 \mathrm{~mm} \mathrm{Hg}$ or not reduced by $20 \%$ below baseline on two consecutive study visits, or IOP of $<6 \mathrm{~mm} \mathrm{Hg}$ or needing further glaucoma drainage surgery, and (b) IOP $>15 \mathrm{~mm} \mathrm{Hg}$ or not reduced by $30 \%$ below baseline on two consecutive study visits, or IOP of $<6 \mathrm{~mm} \mathrm{Hg}$ or needing further glaucoma drainage surgery.

(unpublished unit's trabeculectomy audit). Although the manufacturer has provided some postoperative guidance, there is generally limited experience in the assessment and postoperative management of a 'XEN45 bleb'. Simply applying our usual bleb management in trabeculectomy may not be suitable for XEN45 implant. Further studies should look at whether a less-aggressive bleb management approach produces similar results. Despite such aggressive bleb management in our cohort, we did not find any bleb-related complications such as blebitis, blebitis-associated endophthalmitis, or bleb leak, which have been reported at a rate of $0.5 \%, 0.5 \%$, and $14.0 \%$, respectively, in documented trabeculectomy cases. ${ }^{7}$ It must be noted, however, that these studies had much longer follow-up than our study. The need for bleb intervention in our series does not make XEN45 a 'fit-andforget' procedure similar to other MIGS intervention.
Similar to traditional filtration procedures, XEN45 requires understanding of the conjunctival health and healing, and experience in postoperative bleb assessment and management. It should therefore remain a subspecialist procedure, performed only by surgeons who are experienced in this field.

When we compared the bleb intervention group with the non-intervention group, apart from a higher number of phakic eyes in the bleb intervention group, baseline characteristics, preoperative IOP, or number of preoperative drops were not found to be predictive of the need for needling. The bleb intervention group continued to have higher IOP than the non-intervention group throughout the follow-up visits but this could be due to the inherent nature of the bleb intervention eyes, which are more prone to exuberant wound healing and hence, the higher IOP overall. A significant increase in IOP at month 1 
was observed in the bleb intervention group and not in the non-intervention group. IOP at month 1 may therefore be used as a potential predictive factor to tailor the frequency of postoperative follow-up visits. However, the number in

Table 1b Baseline characteristics of patients who required bleb intervention $v$ s those who did not

\begin{tabular}{|c|c|c|}
\hline & $\begin{array}{l}\text { Non-bleb } \\
\text { intervention } \\
(\mathrm{n}=19)\end{array}$ & $\begin{array}{c}\text { Bleb } \\
\text { intervention } \\
(\mathrm{n}=20)\end{array}$ \\
\hline Mean age & $70.6 \pm 15.4$ & $69.7 \pm 11.9$ \\
\hline Female : male & $1.2: 1$ & $1.3: 1$ \\
\hline \multicolumn{3}{|l|}{ Type of glaucoma } \\
\hline Primary open angle & 14 & 16 \\
\hline Pseudoexfoliation & 2 & 0 \\
\hline $\begin{array}{l}\text { Pigment dispersion } \\
\text { syndrome }\end{array}$ & 0 & 1 \\
\hline Uveitic & 3 & 1 \\
\hline Rubeotic & 0 & 1 \\
\hline Steroid induced & 0 & 1 \\
\hline \multicolumn{3}{|l|}{ Previous surgery } \\
\hline Cataract surgery & 8 & 2 \\
\hline Trabeculectomy & 1 & 2 \\
\hline Phaco-iStent & 0 & 2 \\
\hline \multicolumn{3}{|l|}{ Ocular comorbidities } \\
\hline Dry eyes & 0 & 3 \\
\hline Vein occlusion & 1 & 1 \\
\hline AMD & 0 & 2 \\
\hline Ocular ischaemia & 0 & 1 \\
\hline Mean cup : disc & $0.8(0.7-0.95)$ & $0.8(0.5-0.9)$ \\
\hline $\begin{array}{l}\text { Mean Humphreys visual } \\
\text { field mean deviation }\end{array}$ & $-9.1 \pm 7.2$ & $-5.1 \pm 3.9$ \\
\hline
\end{tabular}

the subgroup analysis in our study was small, and larger studies are required to confirm this finding.

The overall complication rate was low with XEN45 implant. Hypotony was found in $20.5 \%$ of our patients on day 1 . Only one eye required AC reformation at week 3 and by week 4; only one eye had numerical hypotony, which subsequently resolved spontaneously. In contrast, hypotony is a rare complication in other MIGS devices such as Hydrus and iStent, although transient hypotony has been reported in $15.4 \%$ of eyes with Cypass stents. ${ }^{12}$ The national survey of trabeculectomy study reported a much higher rate of early hypotony of $24.3 \%$ within the first 2 weeks of surgery compared to XEN45 implant in our cohort. ${ }^{16}$ Kirwan et $\mathrm{al}^{7}$ also found that $2.6 \%$ of posttrabeculectomy eyes required resuturing of the bleb or revision surgery for significant hypotony in their multicentre series within 3 months of surgery. In addition, the TVT study found persistent hypotony in $13.0 \%$ of patients who had tube surgery and $31.0 \%$ of patients who had trabeculectomy 3 months after the surgery, in contrast to our cohort where late-onset or persistent hypotony was not observed.

We found an initial drop in BCVA on day 1 and month 1 in our cohort. This was most likely attributable to surgically induced inflammation and the use of eye drops in the initial postoperative phase, which resolved by month 3. The two patients who lost more than two lines at the final follow-up visit had uveitis-related complications leading to loss of vision. This is comparable to other MIGS devices but potentially better than trabeculectomy surgeries where $6 \%$ of patients were found to lose more than two Snellen lines at final visit. ${ }^{7}$

Implant blockage was seen in three of our eyes and were treated successfully with Argon laser. This was due

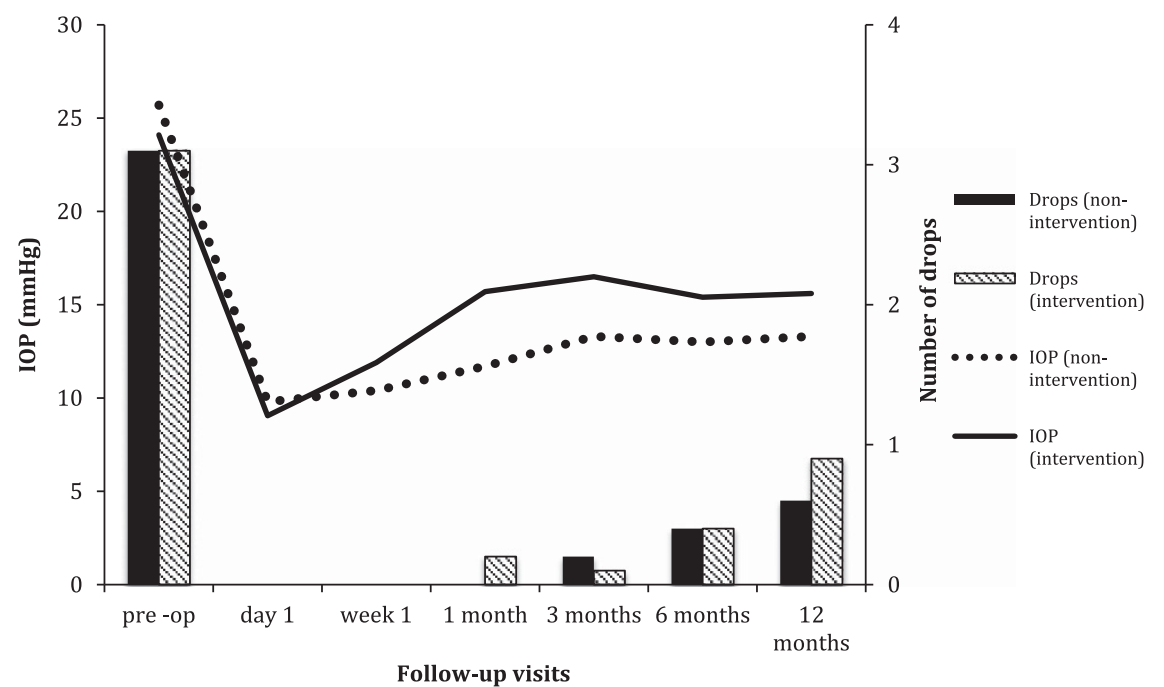

Figure 4 Mean IOP and number of drops in the bleb intervention $v$ s no bleb intervention group over 12-month follow-up visits. 
to poor implantation too close to the iris and we would recommend a careful intraoperative gonioscopy to ensure that the implant is anterior to pigmented trabecular meshwork especially in phakic patients. Stent or tube malposition or obstruction has been reported in $4-18.0 \%$ of cases in iStents, ${ }^{2,3,17} 8.8 \%$ in Cypass, ${ }^{12}$ and $3.0 \%$ in Baerveldt tube. ${ }^{18}$ Only one patient (2.6\%) developed hyphaema in our cohort, compared to $2.3 \%$ in iStent, ${ }^{19} 2.0 \%$ in Baerveldt tube, ${ }^{18}$ and $6-24.6 \%$ in the trabeculectomy studies. ${ }^{7,16,18}$ As the IOP tends to be the lowest immediately post surgery, hyphaema can occur due to the lack of an IOP tamponade. Care should be taken preoperatively with patients taking systemic anticoagulants.

There were some weaknesses in this study. This was a relatively small series with only 1-year follow-up period. We included eyes that were not treatment naive with a varied severity of glaucoma, ranging from mild to advanced neuropathy. Some patients had also undergone previous surgical interventions such as trabeculectomy, iStents, and cataract surgery. Finally, despite an experienced surgeon performing the procedures, there was an inevitable learning curve associated with any new intervention, which might account for the initial complications such as implant blockage and the high needling rates.

In conclusion, we found that XEN45 implant has the potential of achieving a lower final IOP compared to other MIGS devices but is not as effective as the best reported results from modern trabeculectomy. It also comes with a higher complication rate and requires intensive postoperative management compared to other MIGS devices but has a better overall safety profile than trabeculectomy and a shorter surgical time. The large multicentre XEN45 phase IV trial results will hopefully be available in the next few years. The long-term efficacy, cost-effectiveness, and quality-of-life improvement are yet to be established. Further head-tohead trials comparing XEN45 with other MIGS devises and trabeculectomy will be invaluable in guiding clinicians' decision on the surgical management of glaucoma in this modern era.

\section{Summary}

What was known before

- XEN45 is a relatively new device used in minimally invasive glaucoma surgery (MIGS).

- There is not much clinical data on its efficacy and safety profile currently.

\section{What this study adds}

- XEN45 implant has the potential of achieving a lower final intraocular pressure (IOP) compared to other MIGS devices.

- Aggressive bleb management may be required to achieve low IOP.

\section{Conflict of interest}

LA has received research support, honorarium, and travel reimbursement from Glaukos, Aquesys/Allergan, Ivantis, Eyetech care, Thea, and Alcon Pharmaceuticals. The remaining authors declare no conflict of interest.

\section{References}

1 Manasses DT, Au L. The new era of glaucoma micro-stent surgery. Ophthalmol Ther 2016; 5(2): 135-146.

2 Craven ER, Katz LJ, Wells JM, Giamporcaro JEGroup iS. Cataract surgery with trabecular micro-bypass stent implantation in patients with mild-to-moderate open-angle glaucoma and cataract: two-year follow-up. J Cataract Refract Surg 2012; 38(8): 1339-1345.

3 Arriola-Villalobos P, Martínez-de-la-Casa JM, Díaz-Valle D, Fernández-Pérez C, García-Sánchez J, García-Feijoó J. Combined iStent trabecular micro-bypass stent implantation and phacoemulsification for coexistent open-angle glaucoma and cataract: a long-term study. Br J Ophthalmol 2012; 96(5): 645-649.

4 Tan SZ, Au L. Manchester iStent study: 3-year results and cost analysis. Eye (Lond) 2016; 30(10): 1365-1370.

5 Pfeiffer N, Garcia-Feijoo J, Martinez-de-la-Casa JM, Larrosa JM, Fea A, Lemij H et al. A randomized trial of a Schlemm's canal microstent with phacoemulsification for reducing intraocular pressure in open-angle glaucoma. Ophthalmology 2015; 122(7): 1283-1293.

6 Hoeh H, Ahmed II, Grisanti S, Grabner G, Nguyen QH, Rau M et al. Early postoperative safety and surgical outcomes after implantation of a suprachoroidal micro-stent for the treatment of open-angle glaucoma concomitant with cataract surgery. J Cataract Refract Surg 2013; 39(3): 431-437.

7 Kirwan JF, Lockwood AJ, Shah P, Macleod A, Broadway DC, King AJ et al. Trabeculectomy in the 21st century: a multicenter analysis. Ophthalmology 2013; 120(12): 2532-2539.

8 Katz LJ, Erb C, Carceller GA, Fea AM, Voskanyan L, Wells JM et al. Prospective, randomized study of one, two, or three trabecular bypass stents in open-angle glaucoma subjects on topical hypotensive medication. Clin Ophthalmol 2015; 9: 2313-2320.

9 Ahuja Y, Ma Khin Pyi S, Malihi M, Hodge DO, Sit AJ. Clinical results of $\mathrm{ab}$ interno trabeculotomy using the trabectome for open-angle glaucoma: the Mayo Clinic series in Rochester, Minnesota. Am J Ophthalmol 2013; 156(5): 927-935.e922.

10 Pérez-Torregrosa VT, Olate-Pérez Á, Cerdà-Ibáñez M, Gargallo-Benedicto A, Osorio-Alayo V, Barreiro-Rego A et al. Combined phacoemulsification and XEN45 surgery from a temporal approach and 2 incisions. Arch Soc Esp Oftalmol 2016; 91(9): 415-421.

11 Buchacra O, Duch S, Milla E, Stirbu O. One-year analysis of the iStent trabecular microbypass in secondary glaucoma. Clin Ophthalmol 2011; 5: 321-326.

12 Grisanti S, Margolina E, Hoeh H, Rau M, Erb C, KerstenGomez I et al. [Supraciliary microstent for open-angle glaucoma: clinical results of a prospective multicenter study]. Ophthalmologe 2014; 111(6): 548-552.

13 Gandolfi SA, Ungaro N, Ghirardini S, Tardini MG, Mora P. Comparison of Surgical outcomes between canaloplasty and Schlemm's canal scaffold at 24 months' follow-up.

J Ophthalmol 2016; 2016: 3410469. 
14 Edmunds B, Thompson JR, Salmon JF, Wormald RP. The National Survey of Trabeculectomy. II. Variations in operative technique and outcome. Eye (Lond) 2001; 15(Pt 4): 441-448.

15 Gedde SJ, Schiffman JC, Feuer WJ, Herndon LW, Brandt JD, Budenz DL et al. Treatment outcomes in the Tube Versus Trabeculectomy (TVT) study after five years of follow-up. Am J Ophthalmol 2012; 153(5): 789-803. e782.

16 Edmunds B, Thompson JR, Salmon JF, Wormald RP. The National Survey of Trabeculectomy. III. Early and late complications. Eye (Lond) 2002; 16(3): 297-303.
17 Spiegel D, García-Feijoó J, García-Sánchez J, Lamielle H. Coexistent primary open-angle glaucoma and cataract: preliminary analysis of treatment by cataract surgery and the iStent trabecular micro-bypass stent. Adv Ther 2008; 25(5): 453-464.

18 Gedde SJ, Herndon LW, Brandt JD, Budenz DL, Feuer WJ, Schiffman JC et al. Postoperative complications in the Tube Versus Trabeculectomy (TVT) study during five years of follow-up. Am J Ophthalmol 2012; 153(5): 804-814.e801.

19 Patel I, de Klerk TA, Au L. Manchester iStent study: early results from a prospective UK case series. Clin Experiment Ophthalmol 2013; 41(7): 648-652. 